\title{
Weighted Oscillator Strengths and Lifetimes for the Neutral Oxygen Spectrum, O I
}

\author{
I. V. L. Costa ${ }^{1}$, G. H. Cavalcanti ${ }^{2}$, and A. G. Trigueiros ${ }^{1,3}$ \\ ${ }^{1}$ Instituto de Física "Gleb Wataghin", \\ Universidade Estadual de Campinas, UNICAMP, \\ 13083-970 Campinas, São Paulo, Brazil, \\ ${ }^{2}$ Instituto de Física, Universidade Federal Fluminense, UFF \\ Campus da Praia Vermelha-Gragoatá, 24210-310 Niterói, RJ, Brazil, \\ ${ }^{3}$ Instituto de Química, Universidade do Estado do Rio de Janeiro, UERJ, \\ 20550-013 Maracanã, Rio de Janeiro, RJ, Brazil \\ ismael@ifi.unicamp.br,gildo@if.uff.br,andagtri@ifi.unicamp.br
}

Received on 15 June, 2000

\begin{abstract}
The weighted oscillator strengths $(g f)$ and the lifetimes for O I presented in this work were obtained in a multiconfiguration Hartree-Fock relativistic (HFR) approach. In this calculation, the electrostatic parameters were optimized by a least-squares procedure, in order to improve the adjustment to experimental energy levels. This method produces $g f$-values that are in better agreement with intensity observations and lifetime values that are closer to the experimental ones. In this work we present all the experimentally known electric dipole atomic transitions and energy levels for the $\mathrm{O}$ I spectrum.

The data bases, including tables of wavelengths, energy levels, weighted oscillator strenghts and lifetime values for the $\mathrm{O}$ I spectrum, are available in the electronic version of this paper only [on the world wide web, at http://www.sbf.if.usp.br/bjp/Vol31/Num1/].
\end{abstract}

\section{Introduction}

The ground state configuration of the neutral oxygen, $\mathrm{O} \mathrm{I}$, is $1 \mathrm{~s}^{2} 2 \mathrm{~s}^{2} 2 \mathrm{p}^{4}$ with the terms ${ }^{1} \mathrm{~S},{ }^{3} \mathrm{P}$ and ${ }^{1} \mathrm{D}$. The ionization potential for O I is $109837.02 \mathrm{~cm}^{-1}(13.618$ $\mathrm{eV})$.

Edlén [1] has published a detailed analysis of the spectrum of $\mathrm{O} \mathrm{I}$ in which he has revised and extended the earlier work by Fowler [2],Frerichs [3, 4], and More and Rieke [5].

Moore [6] summarized in the book Atomic Energy Levels all the energy levels for O I spectrum. Edlén [7] presented a list of $\mathrm{O}$ I standard lines determined by the Ritz combination principle.

Edlén's [1] classical paper on O I has been revised and extended by Eriksson and Isberg [8,9], Isberg [10]. Huffman et al. [11,12] presented results on absortion spectra: Rydberg series from ground state and metastable states. Moore [13] presented in a new edition of the book Atomic Energy Levels all known levels for the O I. Later, Moore [14] summarized all the energy levels and wavelengths for the $\mathrm{O}$ I spectrum in the book Atomic Energy Levels and Multiplet Table. Kelly [15] summarized all the wavelengths published for
O I in the vacuum ultra-violet, VUV, region.

The purpose of this work is to present a review of all known electric dipole transitions of O I, their oscillator strengths calculated from fitted values of the energy parameters and the lifetimes, calculated by the same method, for all known experimental energy levels. The work we present here was stimulated by the desire to determine weighted oscillator strengths and lifetimes for the O I spectrum. Both parameters are important in the study of laboratory and solar spectra, as oxygen is an astrophysically important element. No extensive source of gf and lifetime values currently exists for this spectrum.

\section{Calculation}

The oscillator strength $f\left(\gamma \gamma^{\prime}\right)$ is a physical quantity related to line intensity $I$ and transition probability $W\left(\gamma \gamma^{\prime}\right)$, as given by Sobelman [16]:

$$
W\left(\gamma \gamma^{\prime}\right)=\frac{2 \omega^{2} e^{2}}{m c^{3}}\left|f\left(\gamma \gamma^{\prime}\right)\right|
$$

with, 


$$
I \propto g W\left(\gamma \gamma^{\prime}\right) \propto g\left|f\left(\gamma \gamma^{\prime}\right)\right|=g f .
$$

Here $\mathrm{m}=$ electron mass, $\mathrm{e}=$ electron charge,$\gamma=$ initial quantum state, $\omega=\left(E(\gamma)-E\left(\gamma^{\prime}\right)\right) / \hbar, E(\gamma)$ initial state energy, $\mathrm{g}=(2 J+1)$ is the number of degenerate quantum states with angular momentum $J$ (in the formula for the initial state). Quantities with primes refer to the final state.

In the equation above, the weighted oscillator strength, $g f$, is given by Cowan [17]:

$$
g f=\frac{8 \pi^{2} m c a_{0}^{2} \sigma}{3 h} S,
$$

where $\sigma=\left|E(\gamma)-E\left(\gamma^{\prime}\right)\right| / h c, h=$ Planck's constant, $\mathrm{c}=$ light velocity, and $a_{0}=$ Bohr radius, and the electric dipole line strength is defined by:

$$
\mathbf{S}=\left|<\lambda J\left\|\mathbf{P}^{1}\right\| \gamma^{\prime} J^{\prime}>\right|^{2} .
$$

This quantity is a measure of the total strength of the spectral line, including all possible transitions between $\mathrm{m}, \quad \mathrm{m}$ ' different $\mathbf{J}_{z}$ eigenstates. The tensor operator $\mathbf{P}^{1}$ (first order) in the reduced matrix element is the classical dipole moment for the atom in units of $e a_{0}$.

To obtain $g f$, we need to calculate $\mathbf{S}$ first, or its square root:

$$
\mathbf{S}_{\gamma \gamma^{\prime}}^{1 / 2}=<\gamma J\left\|\mathbf{P}^{1}\right\| \gamma^{\prime} J^{\prime}>
$$

In a multiconfiguration calculation we have to expand the wavefunction $\mid \gamma J>$ in terms of single configuration wavefunctions, $|\beta J\rangle$, for both upper and lower levels:

$$
\left|\gamma J>=\sum_{\beta} y_{\beta J}^{\gamma}\right| \beta J>.
$$

Therefore, we can have the multiconfigurational expression for $S_{\gamma \gamma^{\prime}}^{1 / 2}$ :

$$
\mathbf{S}_{\gamma \gamma^{\prime}}^{1 / 2}=\sum_{\beta} \sum_{\beta^{\prime}} y_{\beta J}^{\gamma}<\beta J\left\|\mathbf{P}^{1}\right\| \beta^{\prime} J^{\prime}>y_{\beta^{\prime} J^{\prime}}^{\gamma^{\prime}}
$$

The probability per unit time of an atom in a specific state $\gamma J$ to make a spontaneous transition to any state with lower energy is

$$
P(\gamma J)=\sum A\left(\gamma J, \gamma^{\prime} J^{\prime}\right)
$$

where: $A\left(\gamma J, \gamma^{\prime} J^{\prime}\right)$ is the Einstein spontaneous emission transition probability rate, for a transition from the $\gamma J$ state to the $\gamma^{\prime} J^{\prime}$ state. The sum is over all $\gamma^{\prime} J^{\prime}$ states with $E\left(\gamma^{\prime} J^{\prime}\right)<E(\gamma J)$.

The Einstein probability rate is related to $g f$ with the following relation given by Cowan [17]:

$$
g A=\frac{8 \pi^{2} e^{2} \sigma^{2}}{m c} g f
$$

Since the natural lifetime $\tau(\gamma J)$ is the inverse of the probability $P(\gamma J)$, then:

$$
\tau(\gamma J)=\left(\sum A\left(\gamma J, \gamma^{\prime} J^{\prime}\right)\right)^{-1}
$$

The natural lifetime is applicable to an isolated atom. Interaction with matter or radiation will reduce the lifetime of a state.

The values for $g f$ and lifetimes given in Tables 1 and 2 respectively were calculated according to these equations.

In order to obtain better values for oscillator strengths, we calculated the reduced matrix elements $\mathbf{P}^{1}$ by using optimized values of energy parameters which were adjusted from a least-squares calculation, Cowan [17]. In this adjustment, the code tries to fit experimental energy values by varying the electrostatic parameters. This procedure improves $\sigma$ values used in eq. (2) and $y_{\beta J}^{\gamma}$ and $y_{\beta^{\prime} J^{\prime}}^{\gamma^{\prime}}$ values used in eq. (6).

\section{Discussion}

The theoretical predictions for the energy levels of the configurations were obtained by diagonalizing the energy matrices with appropriate HartreeFock relativistic (HFR) values for the energy parameters. For this purpose the computer code developed by Cowan [17] was used. The program allowed us to calculate energy levels, wavelengths, transition probabilities and lifetimes. For the even-parity configurations we have the following picture: $1 \mathrm{~s}^{2} 2 \mathrm{~s}^{2} 2 \mathrm{p}^{4}, 1 \mathrm{~s}^{2} 2 \mathrm{p}^{3}(3 \mathrm{p}+4 \mathrm{p}+5 \mathrm{p}+6 \mathrm{p})$ and $1 \mathrm{~s}^{2} 2 \mathrm{p}^{3}(6 \mathrm{f}+7 \mathrm{f})$. For the odd-parity case we study the $1 s^{2} 2 s^{2} 2 p^{3}(3 s+4 s+5 s+6 s+7 s+8 s+9 s+10 s+11 s+12 s+13 s$ $+14 s+15 s+17 s+19 s+20 s+21 s+22 s+23 s+24 s+25 s+26 s$ $+27 \mathrm{~s}+28 \mathrm{~s}+29 \mathrm{~s}+30 \mathrm{~s}+31 \mathrm{~s}), 1 \mathrm{~s}^{2} 2 \mathrm{~s}^{2} 2 \mathrm{p}^{3}(3 \mathrm{~d}+4 \mathrm{~d}+5 \mathrm{~d}+6 \mathrm{~d}+$ $7 d+8 d+9 d+10 d+11 d+12 d+13 d+14 d+15 d+16 d+17 d+$ $18 \mathrm{~d}+19 \mathrm{~d}+20 \mathrm{~d}+21 \mathrm{~d}+22 \mathrm{~d}+23 \mathrm{~d}+24 \mathrm{~d}+25 \mathrm{~d}+26 \mathrm{~d}+27 \mathrm{~d}+$ $28 \mathrm{~d}+29 \mathrm{~d}+30 \mathrm{~d}$ ), and $1 \mathrm{~s}^{2} 2 \mathrm{~s} 2 \mathrm{p}^{5}$. The members $2 \mathrm{p}^{3} 16 \mathrm{~s}$ and $2 p^{3} 18$ s of the Rydberg serie $2 p^{3} n s$ are unknown. The interpretation of the configuration level structures was made by a least-squares fit of the observed levels. The energy level values were determined from the observed wavelengths by an iterative optimization procedure using the program ELCALC, Radziemsky and Kaufman [18], in which the individual wavelengths are weighted according to their uncertainties. The energy levels adjusted by this method were used to optimize the electrostatic parameters by a least-squares procedure and finally these optimized parameters were used again to calculate the $g f$ - values and lifetimes. This method produces $g$-values that are in better agreement with line intensity observations and lifetimes that are closer to the experimental ones.

We have presented oscillator strengths and lifetimes for all known electric dipole transitions in O I. The 
present work is part of an ongoing program, whose goal is to obtain weighted oscillator strengths and lifetimes for elements of astrophysical importance. The works for Si III, Si V, Si VI, Si VII, Si IX and Si X spectra have been concluded [19], [20], [21], [22], [23], and [24].

In this particular work on $\mathrm{O} I$, the results are part of Costas's research project included in his BSc degree.

All tables are available only in the electronic version of the paper on the world wide web at http://www.sbf.if.usp.br/bjp/Vol31/Num1/.

\section{Acknowledgements}

This work was financially supported by the Fundação de Amparo à Pesquisa do Estado de São Paulo (FAPESP), Brazil, and by Conselho Nacional de Desenvolvimento Científico e Tecnológico (CNPq), Brazil. Computations were carried out at the Prof. John David Rogers Computational Center, UNICAMP.

\section{References}

[1] B. Edlén, Kungl. Svenska Vetenskapsakad. Handl., 20, No. 10, 3 (1943).

[2] A. Fowler, Report on Series in Line Spectra (Fleetway Press London, 1922).

[3] R. Frerichs, Phys. Rev. 34, 1239 (1929).

[4] R. Frerichs, Phys. Rev. 36, 398 (1930).

[5] K. R. More and C. A. Rieke, Phys. Rev. 50, 1054 (1936).

[6] C. E.Moore, Atomic Energy Levels (Nat. Stand. Ref. Data Ser., 467), 1 (Washington: NBS, 1949).

[7] B. Edlén, Rep. Prog. Phys., 26, 181 (1963).

[8] K. B. S. Eriksson and H. B. S. Isberg, Ark. Fys., 24, No. 41, 549 (1963).
[9] K. B. S. Eriksson and H. B. S. Isberg, Ark. Fys., 37, No. 17, 221 (1968).

[10] H. B. S. Isberg, Ark. Fys. 35, No. 40, 495 (1967).

[11] R. E. Huffman, J. C. Larrabee, and Y. Tanaka, J. Chem. Phys., 46, 2213 (1967).

[12] R. E., Huffman, J. C. Larrabee, and Y. Tanaka, J. Chem. Phys., 47, 4462 (1967).

[13] C. E. Moore, Atomic Energy Levels (Nat. Stand. Ref. Data Ser., 35), 1 (Washington: NBS, 1971).

[14] C. E. Moore, Atomic Energy Levels (Nat. Stand. Ref. Data Ser., 3), 7 (Washington: NBS, 1976).

[15] R. L. Kelly, J. Phys. Chem. Ref. Data, 16, 283 (1987).

[16] I. Sobelman, Atomic Spectra and Radiative Transitions (Berlin: Springer, 1979).

[17] R. D. Cowan, The Theory of Atomic Structure and Spectra (Berkeley:University of California Press, 1981).

[18] L. J. Radziemsky and V. Kaufman, J. Opt. Soc. Am., 59, 424 (1969).

[19] F. Callegari and A. G. Trigueiros, ApJS, 119, 181 (1998).

[20] A. G.Trigueiros and C. Jupén, J. Quant. Spectrosc. Radiat. Transfer, 56, 713 (1996).

[21] L. H. Coutinho and A. G. Trigueiros, ApJS, 115, 315 (1998).

[22] L. H. Coutinho and A. G. Trigueiros, ApJS, 121, 591 (1999).

[23] R. V. Orloski, A. G. Trigueiros, and G. H. Cavalcanti, J. Quant. Spectrosc. Radiat. Transfer, 61, 665 (1999).

[24] G. H. Cavalcanti, F. R. T. Luna, and A. G. Trigueiros, J. Quant. Spectrosc. Radiat. Transfer, 64, 5 (2000). 